\title{
Sums of commuting operators with maximal regularity
}

\author{
by \\ Christian Le Merdy and Arnaud Simard (Besançon)
}

\begin{abstract}
Let $Y$ be a Banach space and let $S \subset L_{p}$ be a subspace of an $L_{p}$ space, for some $p \in(1, \infty)$. We consider two operators $B$ and $C$ acting on $S$ and $Y$ respectively and satisfying the so-called maximal regularity property. Let $\mathcal{B}$ and $\mathcal{C}$ be their natural extensions to $S(Y) \subset L_{p}(Y)$. We investigate conditions that imply that $\mathcal{B}+\mathcal{C}$ is closed and has the maximal regularity property. Extending theorems of Lamberton and Weis, we show in particular that this holds if $Y$ is a UMD Banach lattice and $e^{-t B}$ is a positive contraction on $L_{p}$ for any $t \geq 0$.
\end{abstract}

1. Introduction. Let $X$ be a Banach space. Given any $p \in(1, \infty)$ we consider the vector-valued $L_{p}$ space $L_{p}(\mathbb{R} ; X)$ and we let $\mathcal{A}_{X}$ be the derivation operator on $L_{p}(\mathbb{R} ; X)$, defined on its natural domain $W^{1, p}(\mathbb{R} ; X)$. Let $-B$ be the generator of a bounded analytic semigroup on $X$, with domain $D(B)$. We denote by $\mathcal{B}$ the operator on $L_{p}(\mathbb{R} ; X)$ defined by $D(\mathcal{B})=$ $L_{p}(\mathbb{R} ; D(B))$ and $\mathcal{B} u(t)=B(u(t))$ for all $u$ in $D(\mathcal{B})$ and $t$ in $\mathbb{R}$. By definition we say that $B$ has the maximal regularity property $\left(\mathrm{MR}_{\infty}\right.$ for short) if there exists a constant $K>0$ such that

$$
\forall u \in D\left(\mathcal{A}_{X}\right) \cap D(\mathcal{B}), \quad\left\|\mathcal{A}_{X} u\right\|_{p} \leq K\left\|\mathcal{A}_{X} u+\mathcal{B} u\right\|_{p} .
$$

This property implies that for any $T>0$ and any $f \in L_{p}(0, T ; X)$, the Cauchy problem

$$
(\mathrm{CP})_{T}\left\{\begin{array}{l}
u^{\prime}(t)+B u(t)=f(t), \quad t \in(0, T), \\
u(0)=0,
\end{array}\right.
$$

admits a (necessarily unique) solution $u \in W_{0}^{1, p}(0, T ; X) \cap L_{p}(0, T ; D(B))$. It follows e.g. from [4] or [8] that the maximal regularity property $\mathrm{MR}_{\infty}$ for $B$ does not depend on $p \in(1, \infty)$. In 1964 , de Simon [33] showed that if $X$ is a Hilbert space then $\mathrm{MR}_{\infty}$ is satisfied by every negative generator of a bounded analytic semigroup. Then in 1987, Dore and Venni [12] showed that $B$ satisfies $\mathrm{MR}_{\infty}$ if $X$ is a UMD Banach space and if $B$ has bounded imaginary powers, with an estimate $\left\|B^{i s}\right\| \leq K e^{\theta|s|}$ for some $\theta \in(0, \pi / 2)$. Very recently, Kalton and Lancien [15] showed that the latter result does not hold

2000 Mathematics Subject Classification: Primary 47D06; Secondary 47A60, 46M05. 
true if we remove the assumption on imaginary powers. They proved that if $X$ is a separable Banach lattice and if every negative generator of a bounded analytic semigroup on $X$ satisfies $\mathrm{MR}_{\infty}$, then $X$ is isomorphic to a Hilbert space. We refer to [4], [8], [11], and [21] for some background on $\mathrm{MR}_{\infty}$ and its variants, and to [31] for general information on UMD Banach spaces.

We are interested in the following general problem. Let $-B$ and $-C$ be the generators of two commuting bounded analytic semigroups on $X$, and assume that $B$ and $C$ satisfy $\mathrm{MR}_{\infty}$. Which additional conditions ensure that the sum $B+C$ is closed and in that case, does it satisfy $\mathrm{MR}_{\infty}$ as well? Our motivation for this problem lies in results from [13], [30], and [18] which show that in many natural situations, sufficient conditions for $\mathrm{MR}_{\infty}$, such as having bounded imaginary powers or a bounded $H^{\infty}$ functional calculus, are preserved by taking sums of commuting operators. In this paper, we shall obtain positive results in cases when $X$ is a tensor product of two Banach spaces and the operators $B$ and $C$ act on one of the components of the tensor product. A typical situation is that of vector-valued $L_{p}$ spaces. Let $Y$ be a Banach space, let $(\Omega, \mu)$ be a measure space, and let $X=L_{p}(\Omega ; Y)$ for some $p \in(1, \infty)$. Let $B$ and $C$ be negative generators of bounded analytic semigroups on $L_{p}(\Omega, d \mu)$ and $Y$ respectively. It is not hard to see that $B \otimes I_{Y}$ and $I_{L_{p}} \otimes C$ admit closures $\mathcal{B}$ and $\mathcal{C}$ on $X$. In Section 4 (Theorem 4.3), we will show that if $e^{-t B}$ is contractively regular for any $t \geq 0$ (see below for a definition), if $Y$ is a UMD Banach lattice, and if $C$ satisfies $\mathrm{MR}_{\infty}$, then $\mathcal{B}+\mathcal{C}$ is closed and satisfies $\mathrm{MR}_{\infty}$. This result extends a well known result of Lamberton [17] (see also [8, Section 5]), corresponding to the case when $C=0$, and complements some recent work of Weis ([34], [35]). In Section 5 (Theorem 5.2), we will show that if $Y$ is a Hilbert space, if $C$ admits a bounded $H^{\infty}$ functional calculus, and if $B$ satisfies $\mathrm{MR}_{\infty}$, then $\mathcal{B}+\mathcal{C}$ is closed and satisfies $\mathrm{MR}_{\infty}$. This result complements [18, Theorem 1.4].

We will work in the more general context of the so-called vector-valued $S L_{p}$ spaces, and will establish a general result (Theorem 4.1) from which the two theorems presented above will be deduced. For any closed subspace $S \subset L_{p}(\Omega, d \mu)$, called an $S L_{p}$ space, and any Banach space $Y$, we will consider the Banach space $X=S(Y)$, defined as the closure of $S \otimes Y$ in $L_{p}(\Omega ; Y)$, and consider operators $B$ and $C$ acting on $S$ and $Y$ respectively. Theorem 4.1 will provide a general sufficient condition ensuring that the sum $\mathcal{B}+\mathcal{C}$ of the extensions of $B$ and $C$ to $X$ is closed and satisfies $\mathrm{MR}_{\infty}$. Its proof requires several preparatory results of independent interest which are established in the next two sections.

All Banach spaces considered here, including Banach lattices, are complex. Given a Banach space $X$, we denote by $B(X)$ the Banach algebra of all bounded linear operators on $X$. 


\section{A domination principle for contractively regular semigroups.}

Let $1 \leq p \leq \infty$, let $(\Omega, \mu)$ be a measure space, and let $S$ be a closed subspace of $L_{p}(\Omega, d \mu)$. It is plain that for any Banach spaces $Y_{1}, Y_{2}$ and any bounded operator $b: Y_{1} \rightarrow Y_{2}$, the tensor product mapping $I_{S} \otimes b$ extends to a bounded operator from $S\left(Y_{1}\right)$ into $S\left(Y_{2}\right)$, with

$$
\left\|I_{S} \otimes b: S\left(Y_{1}\right) \rightarrow S\left(Y_{2}\right)\right\|=\|b\| .
$$

In particular, given a Banach space $Y$, the tensorization by $I_{S}$ yields an isometric embedding

$$
B(Y) \subset B(S(Y)) \text {. }
$$

The tensorization of a bounded operator on $S$ by $I_{Y}$ requires some special assumptions. We say that a bounded operator $T: S \rightarrow S$ is regular if there exists a constant $K>0$ such that

$$
\forall n \in \mathbb{N}^{*} \forall x_{1}, \ldots, x_{n} \in S, \quad\left\|\sup _{1 \leq i \leq n}\left|T x_{i}\right|\right\|_{p} \leq K\left\|\sup _{1 \leq i \leq n}\left|x_{i}\right|\right\|_{p} .
$$

We denote by $\|T\|_{\mathrm{r}}$ the smallest constant $K$ which satisfies (2.3). Clearly \|\|$_{\mathrm{r}}$ is a norm on the vector space of regular operators on $S$. When $\|T\|_{\mathrm{r}}$ $\leq 1$ we say that $T$ is contractively regular and by extension a contractively regular semigroup $\left(T_{t}\right)_{t \geq 0}$ on $S$ is a $c_{0}$-semigroup such that for all $t \geq 0$, $T_{t}$ is contractively regular. We refer to [29] for some information on regular operators on $S L_{p}$ spaces. This notion extends the well known one of regular operators on $L_{p}$ spaces. We recall that any bounded operator on $L_{1}(\Omega, d \mu)$ or on $L_{\infty}(\Omega, d \mu)$ is regular and that if $p \in(1, \infty)$, a bounded operator $T: L_{p}(\Omega, d \mu) \rightarrow L_{p}(\Omega, d \mu)$ is regular if and only if $T$ is a linear combination of positive operators on $L_{p}(\Omega, d \mu)$ (see e.g. [24] or [32]). In particular we mention that a positive operator $T$ on $L_{p}(\Omega, d \mu)$ satisfies $\|T\|_{\mathrm{r}}=\|T\|$. More generally, $T$ is contractively regular if and only if there exists a positive contraction $\widehat{T}: L_{p}(\Omega, d \mu) \rightarrow L_{p}(\Omega, d \mu)$ such that $|T(f)| \leq \widehat{T}(|f|)$ for every $f \in L_{p}(\Omega, d \mu)$. The following reformulation of regularity will be useful.

Lemma 2.1. Let $T: S \rightarrow S$ be a bounded operator. Then $T$ is regular if and only if for any Banach space $Y$, the tensor product $T \otimes I_{Y}$ extends to a bounded operator on $S(Y)$. Furthermore, we have

$$
\left\|T \otimes I_{Y}: S(Y) \rightarrow S(Y)\right\| \leq\|T\|_{\mathrm{r}} .
$$

Proof. Note that $(2.3)$ means that $\left\|T \otimes I_{\ell_{n}^{\infty}}: S\left(\ell_{n}^{\infty}\right) \rightarrow S\left(\ell_{n}^{\infty}\right)\right\| \leq K$ for any integer $n \geq 1$. Assume that $T$ is regular and let $Y$ be a finite-dimensional Banach space. For any $\varepsilon>0$, there exist an integer $n \geq 1$, a subspace $E \subset \ell_{n}^{\infty}$, and an isomorphism $b: Y \rightarrow E$ such that $\|b\| \cdot\left\|b^{-1}\right\| \leq 1+\varepsilon$. Using (2.1) twice, we obtain $\left\|T \otimes I_{Y}\right\| \leq\|T\|_{\mathrm{r}}(1+\varepsilon)$. Since $\varepsilon$ is arbitrary, we obtain (2.4) for any finite-dimensional $Y$. The inequality for arbitrary $Y$ follows at once because $S \otimes Y$ is dense in $S(Y)$ by definition. Conversely, the boundedness of $T \otimes I_{c_{0}}$ on $S\left(c_{0}\right)$ implies $(2.3)$. 
If $T: S \subset L_{p}(\Omega, d \mu) \rightarrow S$ is regular and $b: Y \rightarrow Y$ is bounded, then $T \otimes b=\left(T \otimes I_{Y}\right)\left(I_{S} \otimes b\right)$ extends to a bounded operator on $S(Y)$ that we denote by $T \bar{\otimes} b$. By (2.1) and (2.4), we have

$$
\|T \bar{\otimes} b: S(Y) \rightarrow S(Y)\| \leq\|T\|_{\mathrm{r}}\|b\| .
$$

If $B$ (resp. $C$ ) is a closed operator on $S$ (resp. $Y$ ), then the tensor product $B \otimes I_{Y}$ (resp. $\left.I_{S} \otimes C\right)$, defined on $D(B) \otimes Y$ (resp. $S \otimes D(C)$ ), is closable on $S(Y)$ (see e.g. [19, Lemma 1]). These closures will be denoted by $\mathcal{B}$ and $\mathcal{C}$.

If $\left(T_{t}\right)_{t \geq 0}$ is a contractively regular semigroup on $S$, then $\left(T_{t} \bar{\otimes} I_{Y}\right)_{t \geq 0}$ is obviously a contraction $\mathrm{c}_{0}$-semigroup on $S(Y)$. It is easy to check that if $-B$ is the generator of $\left(T_{t}\right)_{t \geq 0}$, then $-\mathcal{B}$ is the generator of $\left(T_{t} \bar{\otimes} I_{Y}\right)_{t \geq 0}$. Note that similarly, if $\left(V_{t}\right)_{t \geq 0}$ is a bounded $\mathrm{c}_{0}$-semigroup on $Y$ with generator $-C$, then $\left(I_{S} \bar{\otimes} V_{t}\right)_{t \geq 0}$ is a bounded $c_{0}$-semigroup on $S(Y)$ with generator $-\mathcal{C}$. It should be noticed that if $\left(V_{t}\right)_{t \geq 0}$ extends to a bounded analytic semigroup on $Y$, then the same property holds for $\left(I_{S} \bar{\otimes} V_{t}\right)_{t \geq 0}$ on $S(Y)$.

We now wish to establish a domination principle for contractively regular semigroups on $S$ which will extend a famous inequality of Coifman-Weiss [7, Corollary 4.17]. Our result is also clearly related to [5, Theorem 5.6], and actually extends it. We start with the discrete counterpart of this principle. Let us denote by $\sigma$ the shift operator on $\ell_{p}(\mathbb{Z})$ defined by

$$
\forall\left(x_{n}\right)_{n \in \mathbb{Z}} \in \ell_{p}, \quad \sigma\left[\left(x_{n}\right)_{n \in \mathbb{Z}}\right]=\left(x_{n-1}\right)_{n \in \mathbb{Z}} .
$$

Lemma 2.2. Let $S$ be a closed subspace of $L_{p}(\Omega, d \mu)$ for some $p \in$ $[1, \infty)$. Let $T$ be a contractively regular operator on $S$. Let $Y$ be a Banach space. Then for any sequence $b \in \ell_{1}(\mathbb{N} ; B(Y))$ we have

$$
\left\|\sum_{k \geq 0} T^{k} \bar{\otimes} b(k)\right\|_{B(S(Y))} \leq\left\|\sum_{k \geq 0} \sigma^{k} \bar{\otimes} b(k)\right\|_{B\left(\ell_{p}(\mathbb{Z} ; Y)\right)} .
$$

Proof. Regard $T: S \rightarrow S \subset L_{p}(\Omega, d \mu)$ as having values in $L_{p}(\Omega, d \mu)$. Since $T$ is contractively regular, it admits an extension $\widetilde{T}: L_{p}(\Omega, d \mu) \rightarrow$ $L_{p}(\Omega, d \mu)$ such that $\|\widetilde{T}\|_{\mathrm{r}}=\|T\|_{\mathrm{r}}$. This extension property of regular operators is due to Pisier [29, Theorem 3]. Then for any sequence $b \in \ell_{1}(\mathbb{N} ; B(Y))$ we have

$$
\left\|\sum_{k \geq 0} T^{k} \bar{\otimes} b(k)\right\|_{B(S(Y))} \leq\left\|\sum_{k \geq 0} \widetilde{T}^{k} \bar{\otimes} b(k)\right\|_{B\left(L_{p}(\Omega ; Y)\right)} .
$$

We can now apply Akcoglu's dilation theorem [1] and its generalizations ([6], [26]), which ensure that there exist a measure space $\left(\Omega^{\prime}, \mu^{\prime}\right)$, two contractively regular operators

$$
J: L_{p}(\Omega, d \mu) \rightarrow L_{p}\left(\Omega^{\prime}, d \mu^{\prime}\right) \quad \text { and } \quad P: L_{p}\left(\Omega^{\prime}, d \mu^{\prime}\right) \rightarrow L_{p}(\Omega, d \mu),
$$


and an invertible isometric operator $U: L_{p}\left(\Omega^{\prime}, d \mu^{\prime}\right) \rightarrow L_{p}\left(\Omega^{\prime}, d \mu^{\prime}\right)$ such that both $U$ and $U^{-1}$ are contractively regular, and

$$
\forall n \in \mathbb{N}, \quad \widetilde{T}^{n}=P U^{n} J .
$$

Note by Lemma 2.1 that $\|U\|_{\mathrm{r}} \leq 1$ and $\left\|U^{-1}\right\|_{\mathrm{r}} \leq 1$ imply that $\left\|U^{n} \bar{\otimes} I_{Y}\right\|$ $=1$ for any $n \in \mathbb{Z}$. The Coifman-Weiss transference principle [7] (in fact, a vector-valued version of it) can therefore be applied to the sequence $\left(U^{n} \bar{\otimes} I_{Y}\right)_{n \in \mathbb{Z}}$ and we find that for any sequence $b \in \ell_{1}(\mathbb{Z} ; B(Y))$,

$$
\left\|\sum_{k \in \mathbb{Z}} U^{k} \bar{\otimes} b(k)\right\|_{B\left(L_{p}\left(\Omega^{\prime} ; Y\right)\right)} \leq\left\|\sum_{k \in \mathbb{Z}} \sigma^{k} \bar{\otimes} b(k)\right\|_{B\left(\ell_{p}(\mathbb{Z} ; Y)\right)} .
$$

Assume that $b$ is supported by $\mathbb{N}$. From (2.7), we deduce

$$
\sum_{k \geq 0} \widetilde{T}^{k} \otimes b(k)=\left(P \otimes I_{Y}\right)\left(\sum_{k \geq 0} U^{k} \otimes b(k)\right)\left(J \otimes I_{Y}\right)
$$

on $L_{p}(\Omega, d \mu) \otimes Y$. Since $P$ and $J$ are contractively regular, Lemma 2.1 implies that

$$
\left\|P \bar{\otimes} I_{Y}\right\|_{B\left(L_{p}\left(\Omega^{\prime} ; Y\right), L_{p}(\Omega ; Y)\right)} \leq 1 \quad \text { and } \quad\left\|J \bar{\otimes} I_{Y}\right\|_{B\left(L_{p}(\Omega ; Y), L_{p}\left(\Omega^{\prime} ; Y\right)\right)} \leq 1 .
$$

Therefore (2.6), (2.8) and (2.9) give the desired inequality (2.5).

We shall denote by $\left(U_{t}\right)_{t \geq 0}$ the translation semigroup on $L_{p}(\mathbb{R})$ defined for any $f$ in $L_{p}(\mathbb{R})$ by $U_{t}(f)(s)=f(s-t), s \in \mathbb{R}$. Note that it is obviously a contractively regular semigroup. The following result is a generalization of [5, Theorem 5.6], which we recover when $S=L_{p}(\Omega, d \mu)$, the $T_{t}$ 's are positive contractions, and $b$ is scalar-valued.

THEOREM 2.3. Let $Y$ be a Banach space and $S$ be a closed subspace of $L_{p}(\Omega, d \mu)$ for some $p \in[1, \infty)$. Let $\left(T_{t}\right)_{t \geq 0}$ be a contractively regular semigroup on $S$. Then for any $b \in L_{1}\left(\mathbb{R}_{+} ; B(Y)\right)$ we have

$$
\left\|\int_{0}^{\infty} T_{t} \bar{\otimes} b(t) d t\right\|_{B(S(Y))} \leq\left\|\int_{0}^{\infty} U_{t} \bar{\otimes} b(t) d t\right\|_{B\left(L_{p}(\mathbb{R} ; Y)\right)} .
$$

Proof. We shall only outline the proof. Indeed we follow a well known discretization principle introduced in [7], and whose details appear e.g. in [5, Appendix]. First note that compactly supported functions in $L_{1}\left(\mathbb{R}_{+} ; B(Y)\right)$ are dense in $L_{1}\left(\mathbb{R}_{+} ; B(Y)\right)$, hence we may assume that the support of $b$ is compact. Under this assumption, there exist sequences $b_{N}=\left(b_{N}(k)\right)_{k \geq 0} \in$ $\ell_{1}(\mathbb{N} ; B(Y))$ such that for all $x \in S(Y)$,

$$
\int_{0}^{\infty}\left(T_{t} \bar{\otimes} b(t)\right)(x) d t=\lim _{N \rightarrow \infty} \sum_{k \geq 0}\left(T_{1 / N}^{k} \bar{\otimes} b_{N}(k)\right)(x)
$$

and for any $N \geq 1$, 


$$
\left\|\sum_{k \geq 0} \sigma^{k} \bar{\otimes} b_{N}(k)\right\|_{B\left(\ell_{p}(\mathbb{Z} ; Y)\right)} \leq\left\|\int_{0}^{\infty} U_{t} \bar{\otimes} b(t) d t\right\|_{B\left(L_{p}(\mathbb{R} ; Y)\right)} .
$$

Since $\left\|T_{1 / N}\right\|_{\mathrm{r}} \leq 1$, we can apply Lemma 2.2 to obtain

$$
\left\|\sum_{k \geq 0}\left(T_{1 / N}^{k} \bar{\otimes} b_{N}(k)\right)(x)\right\|_{S(Y)} \leq\left\|\sum_{k \geq 0} \sigma^{k} \bar{\otimes} b_{N}(k)\right\|_{B\left(\ell_{p}(\mathbb{Z} ; Y)\right)}\|x\|_{S(Y)} .
$$

The estimate (2.10) follows from (2.11)-(2.13).

3. Generalized $H^{\infty}$ functional calculus for generators of contractively regular semigroups. $H^{\infty}$ functional calculus for generators of bounded semigroups, or more generally for sectorial operators, was introduced by McIntosh [23] on Hilbert spaces and then developed on general Banach spaces in [10]. Its deep connections with maximal regularity are well known; see e.g. [21] for a survey. Here we shall especially use the so-called generalized $H^{\infty}$ functional calculus introduced in [2]. This approach was already exploited in [18], [19], and [22]. We briefly recall the relevant definitions and refer to the papers quoted above for complements.

For any $\omega \in(0, \pi)$, let $\Sigma_{\omega}$ be the set of all $z \in \mathbb{C}^{*}$ such that $|\operatorname{Arg}(z)|<\omega$. Given a linear operator $A$ on a Banach space $X$, we denote by $D(A), R(A)$, and $N(A)$ the domain, range and kernel of $A$ respectively. We denote by $\sigma(A)$ the spectrum of $A$ and we let $\varrho(A)$ be the resolvent set of $A$. For any $\lambda \in \varrho(A)$, we denote by $R(\lambda, A)=\left(\lambda I_{X}-A\right)^{-1} \in B(X)$ the corresponding resolvent operator. We say that $A$ is sectorial of type $\omega \in(0, \pi)$ if $A$ is closed, densely defined, with the property that $\sigma(A) \subset \overline{\Sigma_{\omega}}$ and

$$
\forall \theta \in(\omega, \pi) \exists C>0 \forall z \in\left(\overline{\Sigma_{\theta}}\right)^{\mathrm{c}}, \quad\|z R(z, A)\| \leq C .
$$

We recall that the negative generator of a bounded $\mathrm{c}_{0}$-semigroup is sectorial of type $\pi / 2$ and that an operator $-A$ is the generator of a bounded analytic semigroup if and only if $A$ is sectorial of type strictly less than $\pi / 2$.

Given a sectorial operator $A$ of type $\omega \in(0, \pi)$ we define its commutant by

$$
E_{A}=\{T \in B(X): \forall \lambda \in \varrho(A), T R(\lambda, A)=R(\lambda, A) T\} .
$$

For any $\theta \in(\omega, \pi)$, we let $H^{\infty}\left(\Sigma_{\theta} ; E_{A}\right)$ be the space of all bounded analytic functions $F: \Sigma_{\theta} \rightarrow E_{A}$. This is a Banach algebra for the norm

$$
\|F\|_{H^{\infty}\left(\Sigma_{\theta} ; E_{A}\right)}=\sup \left\{\|F(z)\|_{B(X)}: z \in \Sigma_{\theta}\right\} .
$$

We then define the (non-closed) subalgebra

$$
\begin{aligned}
& H_{0}^{\infty}\left(\Sigma_{\theta} ; E_{A}\right)=\left\{F \in H^{\infty}\left(\Sigma_{\theta} ; E_{A}\right): \text { there are } s, C>0\right. \text { such that } \\
& \left.\qquad\|F(z)\|_{B(X)} \leq C \frac{|z|^{s}}{(1+|z|)^{2 s}} \text { for } z \in \Sigma_{\theta}\right\} .
\end{aligned}
$$


Let $\omega<\omega^{\prime}<\theta<\pi$, and let $\Gamma_{\omega^{\prime}}$ be the path defined by

$$
\Gamma_{\omega^{\prime}}(t)= \begin{cases}-t e^{i \omega^{\prime}}, & t \in \mathbb{R}_{-}, \\ t e^{-i \omega^{\prime}}, & t \in \mathbb{R}_{+}\end{cases}
$$

Then for any function $F \in H_{0}^{\infty}\left(\Sigma_{\theta} ; E_{A}\right)$ we set

$$
u_{A}(F)=\frac{1}{2 \pi i} \int_{\Gamma_{\omega^{\prime}}} F(\lambda) R(\lambda, A) d \lambda .
$$

Since $A$ is sectorial and $F \in H_{0}^{\infty}\left(\Sigma_{\theta} ; E_{A}\right), u_{A}(F)$ is well defined and belongs to $B(X)$. Furthermore, the definition (3.1) does not depend on the choice of $\omega^{\prime} \in(\omega, \theta)$ and the mapping $u_{A}: H_{0}^{\infty}\left(\Sigma_{\theta} ; E_{A}\right) \rightarrow B(X)$ is an algebra homomorphism. Note that $u_{A}$ is not bounded in general. If we moreover assume that $N(A)=\{0\}$ and $R(A)$ is dense in $X$, then for any $F \in H^{\infty}\left(\Sigma_{\theta} ; E_{A}\right)$ we may define a possibly unbounded operator $u_{A}(F)$ as follows. We let $\varphi$ be the scalar-valued function defined by $\varphi(z)=z /(1+z)^{2}$. Then for $F \in$ $H^{\infty}\left(\Sigma_{\theta} ; E_{A}\right)$, the product function $F \varphi$ belongs to $H_{0}^{\infty}\left(\Sigma_{\theta} ; E_{A}\right)$ and we set

$$
u_{A}(F)=\varphi(A)^{-1} u_{A}(F \varphi) \text {, }
$$

with domain equal to

$$
D\left(u_{A}(F)\right)=\left\{x \in X: u_{A}(F \varphi)(x) \in D(A) \cap R(A)\right\} .
$$

The point here is that the range of $\varphi(A)$ is equal to $D(A) \cap R(A)$ and that the latter space is dense in $X$. Consequently, the operator $u_{A}(F)$ is a closed and densely defined operator, with $D(A) \cap R(A) \subset D\left(u_{A}(F)\right)$. Note that $u_{A}(F)$ is unbounded in general. If $F$ is scalar-valued (i.e. with values in $\left.\operatorname{Span}\left\{I_{X}\right\}\right)$, then the operator $u_{A}(F)$ is simply denoted by $F(A)$.

Let $Y$ be a Banach space. Let $p \in[1, \infty)$, let $S \subset L_{p}(\Omega, d \mu)$ and let $-B$ be the generator of a contractively regular semigroup $\left(T_{t}\right)_{t \geq 0}$ on $S$. We consider the Banach space $X=S(Y)$. Recall that we denote by $\mathcal{B}$ the negative generator of $\left(T_{t} \bar{\otimes} I_{Y}\right)_{t \geq 0}$ on $X$. This operator is then sectorial of type $\pi / 2$. Via the isometric embedding (2.2), we may consider $B(Y)$ as a (closed) subalgebra of the commutant $E_{\mathcal{B}}$; hence for any $\theta>\pi / 2$, we may regard $H_{0}^{\infty}\left(\Sigma_{\theta} ; B(Y)\right)$ as a subalgebra of $H_{0}^{\infty}\left(\Sigma_{\theta} ; E_{\mathcal{B}}\right)$, which allows us to define $u_{\mathcal{B}}(F)$ for any $F \in H_{0}^{\infty}\left(\Sigma_{\theta} ; B(Y)\right)$. Likewise we may regard $B(Y)$ as a subspace of $B\left(L_{p}(\mathbb{R} ; Y)\right)$, which is actually included in the commutant algebra $E_{\mathcal{A}_{Y}}$ of the derivation operator $\mathcal{A}_{Y}$, and we will therefore consider operators $u_{\mathcal{A}_{Y}}(F)$ for $F \in H_{0}^{\infty}\left(\Sigma_{\theta} ; B(Y)\right)$. Note that $\mathcal{A}_{Y}$ is $1-1$ with a dense range.

Theorem 3.1. Let $Y$ be a Banach space. Let $S \subset L_{p}(\Omega, d \mu)$ for some $p \in[1, \infty)$, and let $-B$ be the generator of a contractively regular semigroup on $S$. 
(i) For any $\theta \in(\pi / 2, \pi)$ and any $F \in H_{0}^{\infty}\left(\Sigma_{\theta} ; B(Y)\right)$,

$$
\left\|u_{\mathcal{B}}(F)\right\|_{B(S(Y))} \leq\left\|u_{\mathcal{A}_{Y}}(F)\right\|_{B\left(L_{p}(\mathbb{R} ; Y)\right)} .
$$

(ii) Assume that $B$ is 1-1 with dense range, and let $F \in H^{\infty}\left(\Sigma_{\theta} ; B(Y)\right)$ for some $\theta \in(\pi / 2, \pi)$. If $u_{\mathcal{A}_{Y}}(F)$ is bounded on $L_{p}(\mathbb{R} ; Y)$, then $u_{\mathcal{B}}(F)$ is bounded on $S(Y)$.

Proof. Let $\theta \in(\pi / 2, \pi)$ and let $F \in H_{0}^{\infty}\left(\Sigma_{\theta} ; B(Y)\right)$. We choose $\omega^{\prime} \in$ $(\pi / 2, \theta)$. By the definition of $H_{0}^{\infty}\left(\Sigma_{\theta} ; B(Y)\right)$, the integral $\int_{\Gamma_{\omega^{\prime}}}\|F(\lambda)\|\left|\frac{d \lambda}{\lambda}\right|$ is finite and hence

$$
\int_{\Gamma_{\omega^{\prime}}} \int_{0}^{\infty}\|F(\lambda)\| \cdot\left|e^{\lambda t}\right||d \lambda| d t<\infty .
$$

By Fubini's Theorem, we may therefore define $b \in L_{1}\left(\mathbb{R}_{+} ; B(Y)\right)$ by letting

$$
b(t)=-\frac{1}{2 \pi i} \int_{\Gamma_{\omega^{\prime}}} F(\lambda) e^{\lambda t} d \lambda
$$

and for any $f \in S$ and any $y \in Y$ we have

$$
\begin{aligned}
\int_{0}^{\infty}\left(T_{t} \otimes b(t)\right)(f \otimes y) d t & =-\frac{1}{2 \pi i} \int_{0}^{\infty} \int_{\Gamma_{\omega^{\prime}}} T_{t}(f) \otimes F(\lambda)(y) e^{\lambda t} d \lambda d t \\
& =\frac{1}{2 \pi i} \int_{\Gamma_{\omega^{\prime}}}\left(-\int_{0}^{\infty} T_{t}(f) e^{\lambda t} d t\right) \otimes F(\lambda)(y) d \lambda \\
& =\frac{1}{2 \pi i} \int_{\Gamma_{\omega^{\prime}}}(R(\lambda, B) \otimes F(\lambda))(f \otimes y) d \lambda
\end{aligned}
$$

Applying formula (3.1), we deduce that for any $x \in S \otimes Y$,

$$
u_{\mathcal{B}}(F)(x)=\int_{0}^{\infty}\left(T_{t} \otimes b(t)\right)(x) d t .
$$

Similarly,

$$
u_{\mathcal{A}_{Y}}(F)(x)=\int_{0}^{\infty}\left(U_{t} \otimes b(t)\right)(x) d t .
$$

Indeed, $\mathcal{A}_{Y}$ is the negative generator of $\left(U_{t} \bar{\otimes} I_{Y}\right)_{t \geq 0}$ on $L_{p}(\mathbb{R} ; Y)$. The inequality (3.2) therefore follows from (2.10).

Let us show (ii). We assume that $B$ (hence $\mathcal{B}$ ) is $1-1$ with dense range. We let $\theta \in(\pi / 2, \pi)$ and $F \in H^{\infty}\left(\Sigma_{\theta} ; B(Y)\right)$ and we assume that $u_{\mathcal{A}_{Y}}(F)$ is bounded. We introduce the sequence $\left(\varphi_{n}\right)_{n \geq 1}$ of rational functions defined by

$$
\varphi_{n}(z)=\frac{n^{2} z}{(n+z)(1+n z)} .
$$


Let $x \in X=S(Y)$. Each $F \varphi_{n}$ belongs to $H_{0}^{\infty}\left(\Sigma_{\theta} ; B(Y)\right)$, hence by (i) we have

$$
\left\|u_{\mathcal{B}}\left(F \varphi_{n}\right)(x)\right\|_{S(Y)} \leq\left\|u_{\mathcal{A}_{Y}}\left(F \varphi_{n}\right)\right\|_{B\left(L_{p}(\mathbb{R} ; Y)\right)}\|x\|_{S(Y)} .
$$

For any $n \geq 1, \varphi_{n}(\mathcal{B})(x)$ belongs to $R(\mathcal{B}) \cap D(\mathcal{B})$, hence to $D\left(u_{\mathcal{B}}(F)\right)$, and since $u_{\mathcal{B}}$ is a homomorphism on $H_{0}^{\infty}\left(\Sigma_{\theta} ; B(Y)\right)$, we see that $u_{\mathcal{B}}\left(F \varphi_{n}\right)(x)=$ $u_{\mathcal{B}}(F)\left[\varphi_{n}(\mathcal{B})(x)\right]$. Similarly, $u_{\mathcal{A}_{Y}}\left(F \varphi_{n}\right)=u_{\mathcal{A}_{Y}}(F) \varphi_{n}\left(\mathcal{A}_{Y}\right)$. Consequently,

$$
\begin{aligned}
\| u_{\mathcal{B}}(F)\left[\varphi_{n}(\mathcal{B})\right. & (x)] \|_{S(Y)} \\
& \leq\left\|u_{\mathcal{A}_{Y}}(F)\right\|_{B\left(L_{p}(\mathbb{R} ; Y)\right)}\left\|\varphi_{n}\left(\mathcal{A}_{Y}\right)\right\|_{B\left(L_{p}(\mathbb{R} ; Y)\right)}\|x\|_{S(Y)} .
\end{aligned}
$$

By the sectoriality of $\mathcal{B}$, the sequence $\varphi_{n}(\mathcal{B})$ strongly converges to the identity on $X$ (see e.g. [21]). Again the sectoriality of $\mathcal{A}_{Y}$ implies that the sequence $\left(\varphi_{n}\left(\mathcal{A}_{Y}\right)\right)_{n \geq 0}$ is bounded. Hence the boundedness of $u_{\mathcal{B}}(F)$ follows from $(3.3)$.

We shall deduce two corollaries from Theorem 3.1. If $A$ is a sectorial operator of type $\omega \in(0, \pi)$ on a Banach space $X$, and if $\theta \in(\omega, \pi)$, we say that $A$ admits a bounded $H^{\infty}\left(\Sigma_{\theta}\right)$ functional calculus if there exists a constant $K>0$ such that

$$
\forall F \in H_{0}^{\infty}\left(\Sigma_{\theta}\right), \quad\|F(A)\|_{B(X)} \leq K\|F\|_{H^{\infty}\left(\Sigma_{\theta}\right)} .
$$

We recall (see [23], [10]) that if $A$ is $1-1$ with dense range, then this is equivalent to the property that $F(A)$ is a bounded operator for any $F \in H^{\infty}\left(\Sigma_{\theta}\right)$. It was proved in [9] and [14] that negative generators of contractively regular semigroups on $L_{p}$ spaces $(1<p<\infty)$ admit a bounded $H^{\infty}$ functional calculus. We provide a generalization to subspaces of $L_{p}$ spaces.

Corollary 3.2. Let $p \in(1, \infty)$, let $S \subset L_{p}(\Omega, d \mu)$ be an $S L_{p}$ space, and let $-B$ be the generator of a contractively regular semigroup on $S$. Then for any $\theta>\pi / 2, B$ admits a bounded $H^{\infty}\left(\Sigma_{\theta}\right)$ functional calculus.

Proof. We fix $\theta>\pi / 2$ and apply Theorem 3.1 with $Y=\mathbb{C}$. For any $F \in H_{0}^{\infty}\left(\Sigma_{\theta}\right)$, we have $\|F(B)\| \leq\|F(A)\|$, where $A=\mathcal{A}_{\mathbb{C}}$ is the derivation on $L_{p}(\mathbb{R})$. This operator admits a bounded $H^{\infty}\left(\Sigma_{\theta}\right)$ functional calculus (see $[9]$ ), hence the result follows at once.

REMARK 3.3. If $Y$ is UMD, the operator $\mathcal{A}_{Y}$ admits a bounded $H^{\infty}\left(\Sigma_{\theta}\right)$ functional calculus, hence for any $B$ as in Corollary 3.2 and any UMD Banach space $Y$, the operator $\mathcal{B}$ admits a bounded $H^{\infty}\left(\Sigma_{\theta}\right)$ functional calculus on $S(Y)$.

Corollary 3.4. Let $p \in(1, \infty)$ and let $-B$ be the generator of a contractively regular semigroup on some $S L_{p}$ space $S \subset L_{p}(\Omega, d \mu)$. Let $C$ be an operator on a Banach space $Y$ which satisfies $\mathrm{MR}_{\infty}$. Then the operator $\mathcal{B}+\mathcal{C}: D(\mathcal{B}) \cap D(\mathcal{C}) \rightarrow S(Y)$ is closed, and there exists a constant $K>0$ 
such that

$$
\forall u \in D(\mathcal{B}) \cap D(\mathcal{C}), \quad\|\mathcal{C} u\|_{S(Y)} \leq K\|(\mathcal{B}+\mathcal{C}) u\|_{S(Y)}
$$

Proof. Here we denote by $\mathcal{C}$ the closure of $I_{S} \otimes C$ on $S(Y)$ and to avoid confusion, we denote by $\mathcal{C}_{1}$ the closure of $I_{L_{p}(\mathbb{R})} \otimes C$ on $L_{p}(\mathbb{R} ; Y)$. Since $C$ is sectorial of type $\omega<\pi / 2$, the function $F_{C}: z \mapsto z(z+C)^{-1}$ belongs to $H^{\infty}\left(\Sigma_{\theta} ; B(Y)\right)$ for some $\theta>\pi / 2$. We assume that $C$ satisfies $\mathrm{MR}_{\infty}$, hence applying (1.1), there is a constant $K>0$ such that

$$
\forall v \in D\left(\mathcal{A}_{Y}\right) \cap D\left(\mathcal{C}_{1}\right), \quad\left\|\mathcal{A}_{Y} v\right\|_{L_{p}(\mathbb{R} ; Y)} \leq K\left\|\left(\mathcal{A}_{Y}+\mathcal{C}_{1}\right) v\right\|_{L_{p}(\mathbb{R} ; Y)} .
$$

By [18, Proposition 2.6], this implies that $u_{\mathcal{A}_{Y}}\left(F_{C}\right)$ is bounded. Assume for simplicity that $B$ is $1-1$ with dense range. Then Theorem 3.1(ii) implies that $u_{\mathcal{B}}\left(F_{C}\right)$ is bounded, hence again by [18, Proposition 2.6], we see that $\mathcal{B}+\mathcal{C}$ is closed and that (3.4) holds. When $B$ is not $1-1$ with dense range we can consider the operators $B+\varepsilon I_{S}$ for $\varepsilon>0$, which are invertible and are negative generators of contractively regular semigroups. Then it is easy to check that they satisfy (3.4) with a constant $K$ not depending on $\varepsilon>0$. We conclude by letting $\varepsilon$ tend to 0 .

Remark 3.5. Let $S \subset L_{p}(\Omega, d \mu), Y, B$, and $C$ be as in Corollary 3.4. Then let $\left(T_{t}\right)_{t \geq 0}$ and $\left(V_{t}\right)_{t \geq 0}$ be the semigroups generated by $-B$ and $-C$ on $S$ and $Y$ respectively. It follows from [25, A-I, 3.7] that $\mathcal{B}+\mathcal{C}$ is the negative generator of the semigroup $\left(T_{t} \bar{\otimes} V_{t}\right)_{t \geq 0}$. We may derive two simple properties from this fact. First, if $\mathcal{B}$ is sectorial of type $<\pi / 2$, then the operator $\mathcal{B}+\mathcal{C}$ is sectorial of type $<\pi / 2$ as well. Second, assume that $Y \subset L_{p}\left(\Omega^{\prime}, \mu^{\prime}\right)$ is also an $S L_{p}$ space. Then using Fubini, we may regard $S(Y) \subset L_{p}\left(\Omega \times \Omega^{\prime}, \mu \otimes \mu^{\prime}\right)$ as an $S L_{p}$ space in an obvious way. Assume moreover that $\left(V_{t}\right)_{t \geq 0}$ is contractively regular. Using the identity $S(Y)\left(\ell_{n}^{\infty}\right)=S\left(Y\left(\ell_{n}^{\infty}\right)\right)=\bar{Y}\left(S\left(\ell_{n}^{\infty}\right)\right)$ for any $n \geq 1$, it is easy to check that $\left(T_{t} \bar{\otimes} V_{t}\right)_{t \geq 0}$ is also contractively regular. Thus the assumption that $-B$ and $-C$ generate contractively regular semigroups implies that the same is true for $-(\mathcal{B}+\mathcal{C})$.

\section{A generalization of theorems of Lamberton and Weis on} maximal regularity. For operators $B$ and $C$ as in Corollary 3.4, the next general result gives a sufficient condition under which the sum $\mathcal{B}+\mathcal{C}$ satisfies $\mathrm{MR}_{\infty}$.

THEOREM 4.1. Let $p \in(1, \infty)$, let $S$ be a closed subspace of some $L_{p}(\Omega, d \mu)$ and let $Y$ be a Banach space. Let $-B$ be the generator of a contractively regular semigroup on $S$ and assume that $\mathcal{B}$ is sectorial of type strictly less than $\pi / 2$ and satisfies $\mathrm{MR}_{\infty}$ on $S(Y)$. Then for any operator $C$ on $Y$ satisfying $\mathrm{MR}_{\infty}$, the sum $\mathcal{B}+\mathcal{C}$ is closed and satisfies $\mathrm{MR}_{\infty}$ on $S(Y)$. 
Proof. We let $X=S(Y)$. We know from Corollary 3.4 and Remark 3.5 that $\mathcal{C}+\mathcal{B}$ is closed and sectorial of type strictly less than $\pi / 2$ on $X$. Let $A$ be the derivation operator on $L_{p}(\mathbb{R})$, and let $\Delta=D(A) \otimes D(B) \otimes D(C) \subset$ $L_{p}(\mathbb{R}) \otimes S \otimes Y \subset L_{p}(\mathbb{R} ; X)$. Using the bounded net of mappings $n R(-n, A) \bar{\otimes}$ $n^{\prime} R\left(-n^{\prime}, B\right) \bar{\otimes} n^{\prime \prime} R\left(-n^{\prime \prime}, C\right): L_{p}(\mathbb{R} ; X) \rightarrow \Delta \subset L_{p}(\mathbb{R} ; X)$, for $n, n^{\prime}, n^{\prime \prime} \geq 1$, it is not hard to see that $\mathcal{B}+\mathcal{C}$ satisfies $\mathrm{MR}_{\infty}$ provided that there exists a constant $K>0$ such that for any $u \in \Delta$,

$$
\begin{aligned}
\|\left(A \otimes I_{S}\right. & \left.\otimes I_{Y}\right) u \| \\
& \leq K\left\|\left(A \otimes I_{S} \otimes I_{Y}+I_{L_{p}} \otimes B \otimes I_{Y}+I_{L_{p}} \otimes I_{S} \otimes C\right) u\right\| .
\end{aligned}
$$

Let $\mathcal{B}_{0}$ be the closure of $I_{L_{p}} \otimes B$ on $L_{p}(\mathbb{R} ; S)$. Our assumption that $\mathcal{B}$ satisfies $\mathrm{MR}_{\infty}$ implies that $B$ satisfies $\mathrm{MR}_{\infty}$, hence by Corollary 3.4 and Remark 3.5, $\mathcal{B}_{0}+\mathcal{A}_{S}$ is the negative generator of a contractively regular semigroup on $L_{p}(\mathbb{R} ; S)$. Using the identification $L_{p}(\mathbb{R} ; S)(Y)=L_{p}(\mathbb{R} ; X)$ and applying Corollary 3.4, we deduce that there is a constant $K_{1}>0$ such that for any $u \in \Delta$,

$$
\begin{aligned}
& \left\|\left(A \otimes I_{S} \otimes I_{Y}+I_{L_{p}} \otimes B \otimes I_{Y}\right) u\right\| \\
& \quad \leq K_{1}\left\|\left(A \otimes I_{S} \otimes I_{Y}+I_{L_{p}} \otimes B \otimes I_{Y}+I_{L_{p}} \otimes I_{S} \otimes C\right) u\right\| .
\end{aligned}
$$

We assumed that $\mathcal{B}$ satisfies $\mathrm{MR}_{\infty}$ on $X$, hence we have an estimate $\left\|\left(A \otimes I_{S} \otimes I_{Y}\right) u\right\| \leq K_{2}\left\|\left(A \otimes I_{S} \otimes I_{Y}+I_{L_{p}} \otimes B \otimes I_{Y}\right) u\right\|$ on $\Delta$, whence (4.1) with $K=K_{1} K_{2}$.

Remark 4.2. Let $S, Y, B, C,\left(T_{t}\right)_{t \geq 0}$ and $\left(V_{t}\right)_{t \geq 0}$ be as in Theorem 4.1 and Remark 3.5. Then our Theorem 4.1 says that if $\left(T_{t} \bar{\otimes} I_{Y}\right)_{t \geq 0}$ extends to a bounded analytic semigroup on $S(Y)$ whose negative generator satisfies $\mathrm{MR}_{\infty}$, and if that of $\left(I_{S} \bar{\otimes} V_{t}\right)_{t \geq 0}$ satisfies $\mathrm{MR}_{\infty}$, then the negative generator of the product semigroup $\left(T_{t} \bar{\otimes} V_{t}\right)_{t \geq 0}$ satisfies $\mathrm{MR}_{\infty}$ as well.

We now turn to the special case when $S=L_{p}(\Omega, d \mu)$, with $p \in(1, \infty)$. Let $\left(T_{t}\right)_{t \geq 0}$ be a bounded analytic semigroup on $L_{p}(\Omega, d \mu)$. It was proved by Weis [35, Section 4$]$ that if in addition, $\left(T_{t}\right)_{t \geq 0}$ is a contractively regular semigroup, then its negative generator satisfies $M R_{\infty}$. (In fact Weis only stated this result in the case when the $T_{t}$ 's are positive contractions but his proof works as well in the general case.) Recall that Lamberton [17] had obtained the same conclusion under the assumption that for any $t \geq 0, T_{t}$ extends to contractions from $L_{1}(\Omega, d \mu)$ into itself and from $L_{\infty}(\Omega, d \mu)$ into itself. It should be noticed that Weis's theorem contains Lamberton's as a special case. Indeed, using interpolation (see [3]), it is easy to see that if a linear operator $T: L_{p}(\Omega, d \mu) \rightarrow L_{p}(\Omega, d \mu)$ is both contractive on $L_{1}(\Omega, d \mu)$ and on $L_{\infty}(\Omega, d \mu)$, then $\|T\|_{\mathrm{r}} \leq 1$. It was observed in [8] that Lamberton's Theorem may be extended to $L_{p}(\Omega ; Y)$, provided that $Y$ is any UMD Banach lattice. Here is an extension of these results. 
TheOREM 4.3. Let $Y$ be a UMD Banach lattice, let $(\Omega, \mu)$ be a measure space, and let $p \in(1, \infty)$. Let $\left(T_{t}\right)_{t \geq 0}$ and $\left(V_{t}\right)_{t \geq 0}$ be two bounded analytic semigroups on $L_{p}(\Omega, d \mu)$ and $Y$ respectively. Assume that $\left\|T_{t}\right\|_{r} \leq 1$ for any $t \geq 0$.

(i) $\left(T_{t} \bar{\otimes} I_{Y}\right)_{t \geq 0}$ extends to a bounded analytic semigroup on $L_{p}(\Omega ; Y)$ whose negative generator satisfies $\mathrm{MR}_{\infty}$.

(ii) If the negative generator of $\left(V_{t}\right)_{t \geq 0}$ satisfies $\mathrm{MR}_{\infty}$, then the negative generator of $\left(T_{t} \bar{\otimes} V_{t}\right)_{t \geq 0}$ satisfies $\mathrm{MR}_{\infty}$ as well on $L_{p}(\Omega ; Y)$.

Proof. Clearly (ii) follows from (i), Theorem 4.1, and Remark 4.2 hence we only need to prove (i). We shall use complex interpolation, for which we refer to [3]. Improving an earlier result of Pisier [27], Rubio de Francia [31, Part IIIc] showed the following extrapolation result. Given a UMD Banach lattice $Y$, there exist a Hilbert space $H_{0}$ and a UMD Banach space $Y_{0}$ such that $Y=\left[H_{0}, Y_{0}\right]_{\alpha}$ for some $\alpha \in(0,1)$. We then have

$$
L_{p}(\Omega ; Y)=\left[L_{p}\left(\Omega ; H_{0}\right), L_{p}\left(\Omega ; Y_{0}\right)\right]_{\alpha}
$$

by [3, Theorem 5.1.2]. We let $B$ be the negative generator of $\left(T_{t}\right)_{t \geq 0}$. Then we denote by $\mathcal{B}_{0}, \mathcal{B}_{\alpha}$ and $\mathcal{B}_{1}$ the negative generators of $\left(T_{t} \bar{\otimes} I_{H_{0}}\right)_{t \geq 0},\left(T_{t} \bar{\otimes} I_{Y}\right)_{t \geq 0}$, and $\left(T_{t} \bar{\otimes} I_{Y_{0}}\right)_{t \geq 0}$ respectively.

Assume for a while that these operators are invertible, so that we may consider their imaginary powers. Our goal is to show that

$$
\exists K>0 \exists \theta<\pi / 2 \forall s \in \mathbb{R}, \quad\left\|\mathcal{B}_{\alpha}^{i s}\right\| \leq K e^{\theta|s|} .
$$

Once it is proved, we can conclude as follows. By [30, Theorem 2], this estimate shows that $-\mathcal{B}_{\alpha}$ generates a bounded analytic semigroup on $L_{p}(\Omega ; Y)$. Furthermore $Y$ is a UMD Banach space, hence $L_{p}(\Omega ; Y)$ is UMD as well and so by [12], (4.2) ensures that $\mathcal{B}_{\alpha}$ satisfies $\mathrm{MR}_{\infty}$.

We now proceed to the proof of (4.2). It follows from [16, Corollary 5.2] (and its proof) that $B$ admits a bounded $H^{\infty}\left(\Sigma_{\theta_{0}}\right)$ functional calculus for some $\theta_{0}<\pi / 2$. In particular, there is a constant $K_{0}>0$ such that $\left\|B^{i s}\right\| \leq K_{0} e^{\theta_{0}|s|}$ for any $s \in \mathbb{R}$. Moreover the space $H_{0}$ is a Hilbert space, hence for any $T \in B\left(L_{p}(\Omega)\right)$, the operator $T \otimes I_{H_{0}}$ extends to a bounded operator of norm equal to $\|T\|$ on $L_{p}\left(\Omega ; H_{0}\right)$. Since $\mathcal{B}_{0}^{i s}$ is the closure of $B^{i s} \otimes I_{H_{0}}$ for any $s \in \mathbb{R}$, we obtain

$$
\exists K_{0}>0 \exists \theta_{0}<\pi / 2 \forall s \in \mathbb{R}, \quad\left\|\mathcal{B}_{0}^{i s}\right\| \leq K_{0} e^{\theta_{0}|s|} .
$$

On the other hand, since $Y_{0}$ is UMD the operator $\mathcal{B}_{1}$ admits a bounded $H^{\infty}\left(\Sigma_{\theta}\right)$ functional calculus for any $\theta>\pi / 2$. Indeed, this is implicit in [5]; see also Remark 3.3. In particular,

$$
\forall \theta_{1}>\pi / 2 \exists K_{1}>0 \forall s \in \mathbb{R}, \quad\left\|\mathcal{B}_{1}^{i s}\right\| \leq K_{1} e^{\theta_{1}|s|} .
$$


We then choose $\theta_{1}$ such that

$$
\theta=(1-\alpha) \theta_{0}+\alpha \theta_{1}<\pi / 2
$$

By construction, the imaginary powers $\mathcal{B}_{0}^{i s}, \mathcal{B}_{\alpha}^{i s}$ and $\mathcal{B}_{1}^{i s}$ are compatible, hence by interpolation,

$$
\forall s \in \mathbb{R}, \quad\left\|\mathcal{B}_{\alpha}^{i s}\right\| \leq\left\|\mathcal{B}_{0}^{i s}\right\|^{1-\alpha}\left\|\mathcal{B}_{1}^{i s}\right\|^{\alpha} .
$$

The estimate (4.2) now follows from (4.3)-(4.5), with $K=K_{0}^{1-\alpha} K_{1}^{\alpha}$.

The general case can be deduced as follows. For any $\varepsilon>0$, replace $\left(T_{t}\right)_{t \geq 0}$ by $\left(e^{-\varepsilon t} T_{t}\right)_{t \geq 0}$. Then $\mathcal{B}_{0}, \mathcal{B}_{\alpha}$ and $\mathcal{B}_{1}$ are replaced by $\mathcal{B}_{0}+\varepsilon I, \mathcal{B}_{\alpha}+\varepsilon I$ and $\mathcal{B}_{1}+\varepsilon I$. These operators are invertible, hence the preceding reasoning can be applied to them. The point is that the constants $K_{0}$ and $K_{1}$ appearing in (4.3) and (4.4) can be chosen to be independent of $\varepsilon>0$. Indeed, this follows from the boundedness of the $H^{\infty}$ functional calculi of $\mathcal{B}_{0}$ and $\mathcal{B}_{1}$. Consequently, (4.2) is now replaced by

$$
\exists K>0 \exists \theta<\pi / 2 \forall \varepsilon>0 \forall s \in \mathbb{R}, \quad\left\|\left(\mathcal{B}_{\alpha}+\varepsilon I\right)^{i s}\right\| \leq K e^{\theta|s|} .
$$

Applying [12], we obtain an estimate $\left\|\mathcal{A}_{X} u\right\| \leq K^{\prime}\left\|\mathcal{A}_{X} u+\overline{I_{L_{p}} \otimes \mathcal{B}_{\alpha}} u+\varepsilon u\right\|$ for some constant $K^{\prime}$ only depending on $K, \theta, p$ and $Y$. In particular $K^{\prime}$ does not depend on $\varepsilon>0$, hence we finally get the desired inequality.

REMARK 4.4. It is clear from the above proof that Theorem 4.3 remains true if $Y$ is any UMD Banach space with the property that $Y=[H, Z]_{\alpha}$ for some space $H$ isomorphic to a quotient of a subspace of an $L_{p}$ space (this includes Hilbert spaces), some UMD Banach space $Z$, and some $\alpha \in(0,1)$. This holds in particular if $Y$ is the Schatten $p$-class, for $p \in(1, \infty)$, or more generally a non-commutative $L_{p}$ space for $p \in(1, \infty)$. We do not know if Theorem 4.3 is true for any UMD Banach space $Y$.

5. Maximal regularity on Hilbert-space-valued $L_{p}$ spaces. Let $H$ be a Hilbert space, let $p \in(1, \infty)$, and let $(\Omega, \mu)$ be a measure space. We let $B$ and $C$ be two sectorial operators of type strictly less than $\pi / 2$ on $H$ and $L_{p}(\Omega, d \mu)$ respectively, and denote as usual by $\mathcal{B}$ and $\mathcal{C}$ their extensions to $L_{p}(\Omega ; H)$. We look for conditions under which the sum $\mathcal{B}+\mathcal{C}$ on $L_{p}(\Omega ; H)$ is closed and satisfies $\mathrm{MR}_{\infty}$. It was proved in [18] that this holds true if we assume that $C$ admits a bounded $H^{\infty}\left(\Sigma_{\theta}\right)$ functional calculus on $L_{p}(\Omega, d \mu)$ for some $\theta<\pi / 2$. In Theorem 5.2, we prove that the same result holds if the assumption of bounded $H^{\infty}$ functional calculus is assigned to $B$ (and $C$ satisfies $\left.\mathrm{MR}_{\infty}\right)$.

We fix an orthonormal basis $\left(e_{i}\right)_{i \in I}$ on $H$, for some index set $I$. Let $\left(g_{i}\right)_{i \in I}$ be a family of complex independent Gaussian normal variables on a probability space $\left(\Omega^{\prime}, \mu^{\prime}\right)$. Then we let $S \subset L_{p}\left(\Omega^{\prime}, \mu^{\prime}\right)$ be the closed linear 
span of $\left\{g_{i}: i \in I\right\}$. For any finitely supported family of complex numbers $\left(t_{i}\right)_{i \in I}$, we have $\left\|\sum_{i} t_{i} g_{i}\right\|_{p}=\alpha_{p}\left(\sum_{i}\left|t_{i}\right|^{2}\right)^{1 / 2}$, where $\alpha_{p}$ is the $L_{p}$ norm of any $g_{i}$. Thus the mapping $e_{i} \mapsto \alpha_{p}^{-1} g_{i}$ induces an isometric identification $H=S$, whence

$$
L_{p}(\Omega ; H)=S\left(L_{p}(\Omega, d \mu)\right) .
$$

Lemma 5.1. Any bounded operator $T: S \rightarrow S$ is automatically regular, with $\|T\|=\|T\|_{\mathrm{r}}$.

Proof. When $I$ is finite, this follows from [28, Proposition 3.7] and Lemma 2.1. The general case follows by a simple approximation argument.

Theorem 5.2. Assume that $C$ satisfies $\mathrm{MR}_{\infty}$ on $L_{p}(\Omega, d \mu)$ with $p \in$ $(1, \infty)$. Let $B$ be the negative generator of a bounded analytic semigroup on $H$, which admits a bounded $H^{\infty}$ functional calculus. Then $\mathcal{B}+\mathcal{C}$ is closed and satisfies $\mathrm{MR}_{\infty}$ on $L_{p}(\Omega ; H)$.

Proof. The operator $B$ satisfies $\mathrm{MR}_{\infty}$ (see [33]), hence using the identification $L_{p}\left(\mathbb{R} ; L_{p}(\Omega ; H)\right)=L_{p}\left(\Omega ; L_{p}(\mathbb{R} ; H)\right)$, we see that $\mathcal{B}$ satisfies $\mathrm{MR}_{\infty}$. Let $\left(T_{t}\right)_{t \geq 0}$ be generated by $-B$ on $H$. Since $B$ admits a bounded $H^{\infty}$ functional calculus, it follows from [20, Theorem 4.3] that there exists an invertible operator $R$ on $H$ such that $\left(R T_{t} R^{-1}\right)_{t \geq 0}$ is a contraction semigroup. We let $\mathcal{R}=I_{L_{p}(\Omega, d \mu)} \bar{\otimes} R \in B\left(L_{p}(\Omega ; H)\right)$. Then $\mathcal{R B R}^{-1}$ clearly satisfies $\mathrm{MR}_{\infty}$. Let us identify $H$ with $S \subset L_{p}\left(\Omega^{\prime}, \mu^{\prime}\right)$ as explained above. Then $\left(R T_{t} R^{-1}\right)_{t \geq 0}$ is contractively regular thanks to Lemma 5.1 . It therefore follows from Theorem 4.1 and (5.1) that $\mathcal{R B R} \mathcal{R}^{-1}+\mathcal{C}$ is closed and satisfies $\mathrm{MR}_{\infty}$ on $S\left(L_{p}(\Omega, d \mu)\right)$, hence on $L_{p}(\Omega ; H)$. Since

$$
\mathcal{R B R}^{-1}+\mathcal{C}=\mathcal{R}(\mathcal{B}+\mathcal{C}) \mathcal{R}^{-1},
$$

the result follows at once.

REMARK 5.3. We wish to mention a result which essentially follows from [34] and was indicated to us by Nigel Kalton (in June 2000). Let $X$ be a Banach space and let $-B$ and $-C$ be the generators of two commuting bounded analytic semigroups $\left(T_{t}\right)_{t \geq 0}$ and $\left(V_{t}\right)_{t \geq 0}$ on $X$. Recall from [25, A-I, 3.7] that the product semigroup $\left(T_{t} V_{t}\right)_{t \geq 0}$ is bounded analytic and that its generator is $-(\overline{B+C})$. Kalton's observation is that if $B$ and $C$ satisfy $\mathrm{MR}_{\infty}$ and if $X$ is UMD, then $\overline{B+C}$ satisfies $\mathrm{MR}_{\infty}$. Indeed, since $X$ is UMD, it follows from [34, Theorem 4.2] that there exists $\theta>0$ such that the two sets $\left\{e^{-z B}: z \in \Sigma_{\theta}\right\}$ and $\left\{e^{-z C}: z \in \Sigma_{\theta}\right\}$ are $R$-bounded. Then the "product set" $\left\{e^{-z B} e^{-z C}: z \in \Sigma_{\theta}\right\}$ is $R$-bounded as well. Hence applying [34, Theorem 4.2] again, we deduce that $\overline{B+C}$ satisfies $\mathrm{MR}_{\infty}$. This yields an alternate route to prove the second half of either Theorem 4.3 or Theorem 5.2. We also refer to [16] for recent developments. 


\section{References}

[1] M. Akcoglu, A pointwise ergodic theorem in $L_{p}$-spaces, Canad. J. Math. 27 (1975), 1075-1082.

[2] D. Albrecht, Functional calculi of commuting unbounded operators, Ph.D. thesis, Monash University, Melbourne, 1994.

[3] J. Bergh and J. Löfström, Interpolation Spaces. An Introduction, Springer, New York, 1976.

[4] P. Cannarsa and V. Vespri, On maximal $L_{p}$ regularity for the abstract Cauchy problem, Boll. Un. Mat. Ital. B (6) 5 (1986), 165-175.

[5] P. Clément and J. Pruss, Completely positive measures and Feller semigroups, Math. Ann. 287 (1990), 73-105.

[6] R. Coifman, R. Rochberg and G. Weiss, Applications of transference: the $L_{p}$ version of von Neumann's inequality and the Littlewood-Paley-Stein theory, in: Linear Spaces and Approximation, Birkhäuser, Basel, 1978, 53-67.

[7] R. Coifman and G. Weiss, Transference Methods in Analysis, CBMS Regional Conf. Ser. in Math. 31, Amer. Math. Soc., 1977.

[8] T. Coulhon et D. Lamberton, Régularité $L_{p}$ pour les équations d'évolution, Séminaire d'Analyse Fonctionnelle Paris VI-VII (1984-85), 155-165.

[9] M. Cowling, Harmonic analysis on semigroups, Ann. of Math. 117 (1983), 267-283.

[10] M. Cowling, I. Doust, A. McIntosh and A. Yagi, Banach space operators with a bounded $H^{\infty}$ functional calculus, J. Austral. Math. Soc. Ser. A 60 (1996), 51-89.

[11] G. Dore, $L_{p}$-regularity for abstract differential equations, in: Functional Analysis and Related Topics, Lecture Notes in Math. 1540, Springer, 1993, 25-38.

[12] G. Dore and A. Venni, On the closedness of the sum of two closed operators, Math. Z. 196 (1987), 189-201.

[13] —, - Some results about complex powers of closed operators, J. Math. Anal. Appl. 149 (1990), 124-136.

[14] X. T. Duong, $H_{\infty}$ functional calculus of second order elliptic partial differential operators on $L^{p}$ spaces, in: Miniconference on Operators in Analysis (Sydney, 1989), Proc. Centre Math. Anal. Austral. Nat. Univ. 24, Canberra, 1990, 91-102.

[15] N. Kalton and G. Lancien, A solution to the problem of $L^{p}$-maximal regularity, Math. Z. 235 (2000), 559-568.

[16] N. Kalton and L. Weis, The $H^{\infty}$ calculus and sums of closed operators, preprint, 2000 .

[17] D. Lamberton, Équations d'évolution linéaires associées à des semigroupes de contractions dans les espaces $L_{p}$, J. Funct. Anal. 72 (1987), 252-262.

[18] F. Lancien, G. Lancien and C. Le Merdy, A joint functional calculus for sectorial operators with commuting resolvents, Proc. London Math. Soc. 77 (1998), 387-414.

[19] G. Lancien and C. Le Merdy, A generalized $H^{\infty}$ functional calculus for operators on subspaces of $L_{p}$ and applications to maximal regularity, Illinois J. Math. 42 (1998), 470-480.

[20] C. Le Merdy, The similarity problem for bounded analytic semigroups on Hilbert space, Semigroup Forum 56 (1998), 205-224.

[21] - $H^{\infty}$ functional calculus and applications to maximal regularity, Publ. Math. Besançon 16 (1999), 41-77.

[22] -, Counterexamples on L $L_{p}$-maximal regularity, Math. Z. 230 (1999), 47-62.

[23] A. McIntosh, Operators which have an $H_{\infty}$ functional calculus, in: Miniconference on Operator Theory and Partial Differential Equations (North Ryde, 1986), Proc. Centre Math. Anal. Austral. Nat. Univ. 14, Canberra, 1986, 210-231. 
[24] P. Meyer-Nieberg, Banach Lattices, Springer, Berlin, 1991.

[25] R. Nagel, One-Parameter Semigroups of Positive Operators, Lecture Notes in Math. 1184, Springer, 1986.

[26] V. Peller, Isometric dilation of contraction, approximation by isometries, and von Neumann's inequality in an $L_{p}$ space, Math. Inst. Steklov (LOMI) Leningrad (1978).

[27] G. Pisier, Some applications of the complex interpolation method to Banach lattices, J. Anal. Math. 35 (1979), 264-281.

[28] - Factorization of Linear Operators and Geometry of Banach Spaces, CBMS Regional Conf. Ser. in Math. 60, Amer. Math. Soc., 1986.

[29] —, Complex interpolation and regular operators between Banach lattices, Arch. Math. (Basel) 62 (1994), 261-269.

[30] J. Pruss and H. Sohr, On operators with bounded imaginary powers in Banach spaces, Math. Z. 203 (1990), 429-452.

[31] J. L. Rubio de Francia, Martingale and integral transforms of Banach space valued functions, in: Probability and Banach Spaces, Lecture Notes in Math. 1221, Springer, 1986, 195-222.

[32] H. H. Schaefer, Banach Lattices and Positive Operators, Springer, Berlin, 1974.

[33] L. de Simon, Un'applicazione della teoria degli integrali singolari allo studio delle equazioni differenziali lineare astratte del primo ordine, Rend. Sem. Mat. Univ. Padova 34 (1964), 205-223.

[34] L. Weis, Operator-valued Fourier multiplier theorems and maximal $L_{p}$ regularity, Math. Ann. 319 (2001), 735-758.

[35] - A new approach to maximal $L_{p}$-regularity, in: Evolution Equations and their Applications in Physical and Life Sciences (Bad Herrenalb, 1998), G. Lumer and L. Weis (eds.), Lecture Notes in Pure and Appl. Math. 215, Dekker, 2001, 195-214.

Département de Mathématiques

Université de Franche-Comté

25030 Besançon Cedex, France

E-mail: lemerdy@math.univ-fcomte.fr

simard@math.univ-fcomte.fr

Received February 14, 2000

Revised version March 26, 2001 